\title{
Predicting unplanned medical visits among patients with diabetes: translation from machine learning to clinical implementation
}

\author{
Arielle Selya ${ }^{1,2,3,4^{*}} \mathbb{D}$, Drake Anshutz ${ }^{2,5}$, Emily Griese ${ }^{2,6}$, Tess L. Weber ${ }^{2}$, Benson Hsu ${ }^{3}$ and Cheryl Ward ${ }^{2,7}$
}

\begin{abstract}
Background: Diabetes is a medical and economic burden in the United States. In this study, a machine learning predictive model was developed to predict unplanned medical visits among patients with diabetes, and findings were used to design a clinical intervention in the sponsoring healthcare organization. This study presents a case study of how predictive analytics can inform clinical actions, and describes practical factors that must be incorporated in order to translate research into clinical practice.
\end{abstract}

Methods: Data were drawn from electronic medical records (EMRs) from a large healthcare organization in the Northern Plains region of the US, from adult ( $\geq 18$ years old) patients with type 1 or type 2 diabetes who received care at least once during the 3-year period. A variety of machine-learning classification models were run using standard EMR variables as predictors (age, body mass index (BMI), systolic blood pressure (BP), diastolic BP, low-density lipoprotein, high-density lipoprotein (HDL), glycohemoglobin (A1C), smoking status, number of diagnoses and number of prescriptions). The best-performing model after cross-validation testing was analyzed to identify strongest predictors.

Results: The best-performing model was a linear-basis support vector machine, which achieved a balanced accuracy (average of sensitivity and specificity) of $65.7 \%$. This model outperformed a conventional logistic regression by 0.4 percentage points. A sensitivity analysis identified BP and HDL as the strongest predictors, such that disrupting these variables with random noise decreased the model's overall balanced accuracy by 1.3 and 1.4 percentage points, respectively. These recommendations, along with stakeholder engagement, behavioral economics strategies, and implementation science principles helped to inform the design of a clinical intervention targeting behavioral changes.

Conclusion: Our machine-learning predictive model more accurately predicted unplanned medical visits among patients with diabetes, relative to conventional models. Post-hoc analysis of the model was used for hypothesis generation, namely that $\mathrm{HDL}$ and $\mathrm{BP}$ are the strongest contributors to unplanned medical visits among patients with diabetes. These findings were translated into a clinical intervention now being piloted at the sponsoring healthcare organization. In this way, this predictive model can be used in moving from prediction to implementation and improved diabetes care management in clinical settings.

\footnotetext{
*Correspondence: Arielle.Selya@SanfordHealth.org

2 Behavioral Sciences Group, Sanford Research, 2301 East 60th Street

North, Sioux Falls, SD 57104, USA

Full list of author information is available at the end of the article
} original author(s) and the source, provide a link to the Creative Commons licence, and indicate if changes were made. The images or other third party material in this article are included in the article's Creative Commons licence, unless indicated otherwise in a credit line to the material. If material is not included in the article's Creative Commons licence and your intended use is not permitted by statutory regulation or exceeds the permitted use, you will need to obtain permission directly from the copyright holder. To view a copy of this licence, visit http://creativecommons.org/licenses/by/4.0/. The Creative Commons Public Domain Dedication waiver (http://creativeco mmons.org/publicdomain/zero/1.0/) applies to the data made available in this article, unless otherwise stated in a credit line to the data. 
Keywords: Diabetes, Unplanned medical visits, Machine learning, Predictive model

\section{Background}

There are approximately 1.5 million new diabetes diagnoses among people 18 years and over every year, and in 2018 , approximately 34.2 million persons $(10.5 \%)$ in the US had diabetes [1]. In 2017, 83,564 deaths were attributed to diabetes in the United States, and that year, diabetes was the 7th leading cause of death in the United States (25.7 deaths per 100,000 population) [1].

Diabetes imposes significant healthcare utilization and costs [2]. Americans with diabetes in 2017 spent approximately $\$ 16,700$ annually in health care costs, 2.3 times higher than those without diabetes [3]. Total costs of diabetes in 2017 were $\$ 327$ billion annually, of which $\$ 237$ billion were in direct medical costs [3]. In addition, there is a positive relationship between lack of health insurance and prevalence of diagnosed diabetes, exacerbating the risks for uninsured Americans [4]. By 2034, the population with diabetes is expected to increase by $100 \%$ and the cost is expected to increase by $53 \%$ [5].

Patients with diabetes generally have increased healthcare utilization, including planned visits (e.g. clinic visits, outpatient departments), as well as unplanned visits (e.g. emergency department and urgent care visits), compared to those without diabetes [3, 6]. The 2011 National Health Interview Survey Diabetes revealed that 30\% of diabetic patients had at least one emergency department visit within the last year, compared to only $20 \%$ of the general population [6]. The majority of emergency department visits among patients with diabetes are likely related to acute glycemic complications (hyperglycemia and hypoglycemia) [6]; however, most adults with diabetes have at least one comorbid chronic condition [7] which could contribute to these visits as well. Unplanned visits typically present a greater burden to patients and insurers due to the higher cost of these visits.

Additionally, social and behavioral factors are associated with unplanned medical visits among the population of patients with diabetes. Lower socioeconomic status, longer disease duration, disease severity, and co-morbid depression are all significant determinants of unplanned medical visits and hospitalizations [8]. More precisely, patients with diabetes with very high current depressive symptoms were two times more likely to have an unplanned emergency department visit, and patients who were diagnosed more than 10 years ago were 1.3 times as likely to have an unplanned emergency department visit [8]. Additionally, cigarette smoking is associated with a greater likelihood of unplanned medical visits [9]. However, unplanned visits remain a high-impact problem for patients and healthcare systems alike, highlighting the need for improved prediction models that can be implemented clinically.

Because of the increased risks and associated costs for patients with diabetes, there is a significant need to improve prediction capabilities aimed at reducing unplanned medical visits for this group of patients. A majority of medical risk prediction models have been developed using stepwise logistic regression, while machine learning classification methods have been largely unexplored [10]. Machine learning methods offer the additional possibility to improve prediction based on pattern detection of many variables simultaneously, as has been shown in applications on predicting obesity [11] and compliance with dietary recommendations [12], predicting metabolic syndrome from physical characteristics and lab results [13], identifying binge drinkers from parenting variables [14] and drinking motives [15], and predicting high blood pressure using body measures [16]. The current study utilizes electronic medical record (EMR) data from a large healthcare system and develops a machine learning based predictive model to predict any versus no unplanned medical visits over a 3-year period among adult patients with diabetes. We also discuss how the findings of this predictive model were translated into a clinical intervention currently underway at the sponsoring healthcare organization.

\section{Methods \\ Sample}

Data were obtained from electronic medical records (EMRs) in EPIC from Sanford Health, a not-for-profit rural healthcare system that primarily serves South Dakota, North Dakota, Northern and Southwest Minnesota, Northwest Iowa, and parts of Nebraska. Sanford Health includes roughly 44 hospitals, 1382 physicians and 9703 nurses delivering care in more than 80 specialty areas. All data were de-identified according to the Health Insurance Portability and Accountability Act HIPAA deidentification method Safe Harbor $\mathbb{1}$ 164.514(b)(2). The dataset included records from all patients who visited a Sanford healthcare facility between January 1, 2014 and December 30, $2016(N=1,143,028)$. Only adult patients (age $\geq 18 ; N=875,168$ ) with a diagnosis of diabetes (ICD10 codes E10.xx and E11.xx; $N=67,575)$ were included in the current study. Further, only patients who reported a residential zip code in Minnesota (MN), North Dakota (ND), or South Dakota (SD) were included in the current study $(N=63,781)$, due to low sample sizes in other 
states. Finally, patients who had missing data on the outcome variable of unplanned medical visits or any of the predictor variables were excluded, for a final sample size of $N=43,831$.

\section{Measures}

The outcome was any versus no unplanned medical visits during the 3-year period over which EMR data were collected. This outcome was derived from four separate variables: emergency department visits, hospitalizations, hospital observations, and urgent care visits. All four types of visits were summed and dichotomized as $\geq 1$ versus 0 unplanned medical visits.

Predictor variables included all numeric variables that were common and readily available in Sanford's EMRs. Ten variables were selected and are described in detail below.

Age was measured in years at time of initial analyses $(12 / 1 / 2016)$.

Body mass index (BMI) was obtained from EMRs as $\mathrm{kg} / \mathrm{m}^{2}$. Extreme values $(<15$ or $>60)$ were assumed to be errors and were set to missing. Values from the most recent visit in the 3-year period were used, as this was the only measure in the dataset provided by the sponsoring healthcare organization (see Limitations).

Blood pressure (BP) was obtained in $\mathrm{mm} / \mathrm{Hg}$. Values from the most recent visit in the 3-year period were used, as this was the only measure in the dataset provided by the sponsoring healthcare organization (see Limitations). Systolic BP and diastolic BP were included as two separate variables.

Serum cholesterol was obtained as both low-density lipoprotein (LDL) and high-density lipoprotein (HDL) in $\mathrm{mg} / \mathrm{dL}$. Extreme values in HDL $(<10$ or $>100)$ or LDL $(<20$ or $>200)$ were assumed to be errors and were set to missing. Values from the most recent laboratory result were used, as this was the only measure in the dataset provided by the sponsoring healthcare organization (see Limitations). LDL and HDL were analyzed as two separate variables.

Glycohemoglobin (A1C) was measured from the most recent laboratory result, as this was the only measure in the dataset provided by the sponsoring healthcare organization (see Limitations). A1C values below 4 or above 15 were assumed to be errors and were set to missing.

Ranked smoking status was obtained by patient selfreport as a vital sign on their most recent visit, as this was the only measure in the dataset provided by the sponsoring healthcare organization (see Limitations). A ranked variable was created as follows from the several possible response categories, with higher values indicating more smoke exposure: never smoker (0), passive smoker (1), former smoker (2), current some day smoker (3), current everyday smoker, light tobacco smoker, or heavy tobacco smoker (4).

Number of diagnoses on "problem list" was derived from the most recently available list over the 3-year period.

Number of prescriptions were aggregated over the 3 -year period and was used as a numeric variable.

\section{Analyses \\ Machine learning}

All analyses predicted the unplanned medical visit status of each patient (i.e., which patients had at least one versus no unplanned medical visits in the 3-year period), and this classification task was based on the 10 EMR variables above (age, BMI, systolic and diastolic BP, HDL and LDL cholesterol, A1C, ranked smoking status, number of diagnoses on the patient's "problem list," and the number of prescriptions in the 3-year period). Four types of machine learning were utilized: discriminant analysis (linear and quadratic), support vector machines (SVM; linear basis and radial basis), single-layer artificial neural nets (NN's) triple-layer deep nets (DNN's), and extreme gradient boosting (XG boost). R software [17] was used for all analyses, including the packages MASS for discriminant analysis [18], e1071 for SVM's [19], nnet for single-layer NN's [18], deepnet for triple-layer DNN's [20], and xgboost for XG boost [21]. A logistic regression was run for purposes of comparing machine learning results with conventional prediction approaches. All R code for this project is publicly available on github at: https:// github.com/ArielleSelya/Diabetes-Predictive-Model.

\section{Cross-validation testing}

Since classifiers are susceptible to overtraining (i.e. when the classifier can predict the training dataset with high accuracy, but fits noise and thus has not learned patterns that generalize to other datasets), cross-validation testing is important to identify models that have detected patterns that are truly important in the prediction task. Cross-validation testing is performed by partitioning all available data points into a training set and a testing set; the classifier is trained on the data from the training set, and the generalization of the prediction task learned by the classifier is tested using the data from the testing set. Nested cross-validation is a procedure which further reduces overfitting by performing this cross-validation procedure on a subset of the data ("inner fold," which is then split into training and test sets), selecting the optimal parameters, and testing on the remaining data in the "outer fold." This procedure is important in selecting optimal hyperparameters for the model, as using the same dataset for generalization as well as model selection introduces bias [22]. 
In particular for this study, five-fold cross-validation was used. For models with hyperparameters to be optimized (i.e. SVM, NN, DNN, XG boost), nested crossvalidation was used to select optimize hyperparameters. Five-fold cross-validation was used on the outer loop. Inside each outer loop, the possible hyperparameters were looped over, with balanced accuracy for each hyperparameter being evaluated by another "inner" loop of five-fold cross-validation. The hyperparameters and balanced accuracy for each outer fold were recorded, and the most common set of hyperparameters were selected as the "final" model.

Both training performance (prediction on the training dataset) and generalization performance (prediction on the testing set) were assessed using confusion matrices. SVM, NN, DNN, and XG boost classifiers were optimized by running several iterations over different parameter values. For SVM, possible cost parameters were 0.1, $0.5,1,5,10,25$, and 50; and for radial SVM, possible gamma parameters were 0.0001, 0.001, 0.003, 0.007, 0.1, 0.5 , and 1 . For single-layer NN's, possible hidden layer sizes were $1,2,5,10,15$, and 20 ; the possible maximum training iterations were 100,150 , and 200; and the possible decay parameters were $0,0.1,0.3,0.5$, and 0.9. For triple-layer NN's, possible sizes of the first hidden layer were $1,5,10,15$, and 20; for the second and third hidden layers, possible values were $1,5,10$, and 20; possible learning rates were $0,0.1,0.5$, and 1 ; possible momentum values of the learning rate were $0,0.1,0.5$, and 1 ; and possible numbers of training iterations were 10 and 20. For XG boost, possible maximum depth values were $3,6,10$, 15 , and 20; possible eta values (learning rate) were 0.01 , $0.5,0.1,0.3$, and 0.6 ; possible values for number of rounds were $50,100,150$, and 200; possible gamma values were $0,0.5,1,5,10$, and 25 ; and possible ratios of columns variables per tree were $0.1,0.5$, and 1.0. For each classifier, the model with the highest performance (see next section) is reported.

\section{Performance metric}

Many performance metrics exist for classifiers, and we selected one for the current application for the following reasons. First, class imbalance in a dataset (here, 57\% of the sample with unplanned medical visits vs. $43 \%$ without) can impact classifier performance, such that the classifier may show bias towards the more common class. Since the conventional definition of overall model accuracy $\left(\frac{1-\text { incorrect predictions }}{\text { total predictions }}\right)$ is sensitive to class imbalance, the imbalance would need to be taken into account if using this traditional accuracy metric. However, using this accuracy metric tends to result in overprediction of positives, often at the expense of a high false-alarm rate; this is undesirable in clinical settings due to the cost and potential harm resulting from false alarms (i.e. providing interventions or treatment to patients who do not need it). For example, strict criteria for prostate cancer screening have historically erred on the side of identifying positives (both true and false), resulting in high rates of unnecessary biopsies and other treatments for patients who (as is now understood) were unlikely to ever show clinical symptoms of prostate cancer. Thus, given the clinical applications of the current study, it is essential to maximize both the true positive (sensitivity) and true negative (specificity) rates. Previous work has shown that maximizing the sum of sensitivity and specificity is appropriate for clinical applications with trade-offs between accurate risk detection and minimizing false alarms [23]. Here, we use such a measure: $\frac{\text { sensitivity+specificity }}{2}$ where we divide by 2 in order for more intuitive interpretation, i.e. to average across the correct predictions within each class. This metric, "balanced accuracy," has the advantage of selecting a model based on the maximum true positive and true negative rates (i.e. minimizing both false positives and false negatives), which is appropriate for clinical applications [23].

Since balanced accuracy contains two possible categories and averages sensitivity and specificity, chance performance is $50 \%$. Notably, this cutoff holds even in cases of imbalance (here, the $57 \%$ default sensitivity would be balanced by the $43 \%$ specificity, resulting in a performance of 50\%). Classifier performance versus chance was measured using a binomial test of the success rate out of the 1000 cross-validation iterations.

\section{Sensitivity testing}

In order to derive clinical implications from the predictive model, it is valuable to know which variables are most strongly predictive of unplanned medical visits. Although being important for prediction does not necessarily indicate causality, many of the modifiable predictors (A1C, BMI, BP, cholesterol, smoking) do have plausible causal effects on diabetes and its complications. Thus, in order to determine the modifiable variables that are most strongly indicative of unplanned medical visits, a variant of sensitivity testing was performed: for one variable at a time, random noise was added to that variable. These random values were drawn from a normal distribution with the same mean as the variable being tested, and a standard deviation of $30 \%$ that of that variable. For the sensitivity testing, cross-validation was done using 25 iterations of hold-20\%-out repeated subsampling; this cross-validation procedure was different from the approach used in the main analysis due to the greater need for precision introduced by adding noise. These were compared to the base-case balanced accuracy using the original dataset and the classifier with the 
optimal hyperparameters obtained above (which was rerun using this cross-validation method, for comparability). Larger disruptions to the balanced generalization accuracy as a result of disrupting the information content of that variable (i.e. by adding noise) indicates a greater importance of that variable to the prediction task, and potentially as a clinical target for intervention.

\section{Clinical intervention development}

The process by which the above findings were incorporated into a clinical intervention are discussed, along with other factors including patient and physician engagement, interfacing with clinical operations, and decisionmaking under real-world practical limitations.

\section{Results}

Table 1 shows the characteristics of the sample, summarized by patients who did versus did not have unplanned medical visits during the 3-year period. Patients with at least one unplanned visit tended to be slightly older (66 vs. 65 years old), rank higher on the smoking scale (2 vs. 1 ), have more diagnoses on the problem list ( 4 vs. 3 ), have lower HDL values (42 vs. $44)$, and have been prescribed considerably more medications over the 3 -year period ( 205 vs. 88 ) (all $p<0.05$ ). The two groups had similar mean levels of diastolic blood pressure, but those with at least one unplanned visit had a wider interquartile range (IQR: $64-80$ vs. 66-80), resulting in a statistically significant difference. Similarly, the two groups had similar mean levels of A1C, but those with at least one unplanned visit had a wider IQR (6.3-7.9 vs. 6.3-7.8), resulting in a significantly different difference. Though these differences are minor, they are statistically significant in part because of the large sample size. The significance should be interpreted along with the effect size; the differences reported here are unlikely to be clinically meaningful. No significant difference was observed for BMI, systolic blood pressure or LDL cholesterol $(p>0.05)$.

Table 2 shows the balanced accuracy of each type of classifier after optimization (i.e. using optimal parameter settings), averaged across the 1000 cross-validation runs. Logistic regression (bottom row) is intended as a comparison, as it only models main effects of each predictor and does not contain any interaction terms. Logistic regression performed reasonably well, with a sensitivity (true positive rate) of $70.2 \%$ and a specificity (true negative rate) of $60.4 \%$. XG boost classifiers found the highest sensitivity of all models (83.3\%), but specificity was low (33.9\%); thus, this was considered a low performing model, especially relative to logistic regression, due to its inability to distinguish between classes. For similar reasons, linear discriminant analysis also resulted in a low performing model, with high sensitivity (75.2\%) and low specificity (50.8\%). Linear SVM was the only model that outperformed the logistic regression, at $65.7 \%$ balanced accuracy, with the sensitivity (60.2\%) and specificity $(71.1 \%)$ both being significantly above chance, and significantly higher than logistic regression $(p=0.03$ according to a t-test of the balanced accuracy). Single and triple hidden layer neural networks (NN's and DNN's, respectively) were found to underperform in comparison to logistic regression balanced accuracy, and were also highly variable across cross-validation folds, as were XG boost classifiers. Table 3 shows the best-performing hyper-parameters within each outer fold.

Table 4 shows the corresponding balanced accuracy for training sets for the optimized classifiers shown in Table 2. Balanced accuracies are extremely similar across training and testing accuracies (different by only

Table 1 Characteristics of patients with diabetes by unplanned visit status

\begin{tabular}{|c|c|c|c|}
\hline Predictor variable & $\begin{array}{l}\text { No unplanned visits } \\
(N=18,771)\end{array}$ & $\begin{array}{l}\geq 1 \text { Unplanned visits } \\
(N=25,060)\end{array}$ & $p$ value \\
\hline Age & $65(55-74)$ & $66(55-76)$ & $<.0001$ \\
\hline BMI & $32.3(28.3-37.0)$ & $32.2(28.0-37.3)$ & $=.2454$ \\
\hline Systolic BP & $126.0(118.0-134.0)$ & $126.0(116.0-136)$ & $=.0089$ \\
\hline Diastolic BP & $72.0(66.0-80.0)$ & $72.0(64.0-80.0)$ & $<.0001$ \\
\hline LDL cholesterol & $85.0(67.0-106.0)$ & $84.0(65.0-106.0)$ & $=.0053$ \\
\hline HDL cholesterol & $44.0(37.0-53.0)$ & $42.0(35.0-52.0)$ & $<.0001$ \\
\hline $\mathrm{A} 1 \mathrm{C}$ & $6.9(6.3-7.8)$ & $6.9(6.3-7.9)$ & $=.0001$ \\
\hline Ranked smoking status & $1.0(0.0-2.0)$ & $2.0(0.0-2.0)$ & $<.0001$ \\
\hline Number of diagnoses on problem list & $3.0(2.0-4.0)$ & $4.0(3.0-6.0)$ & $<.0001$ \\
\hline Number of prescriptions & $88.0(40.0-179.0)$ & $205.0(96.0-408.0)$ & $<.0001$ \\
\hline
\end{tabular}

Variables are summarized as median (interquartile range). $A 1 C$ glycohemoglobin. $B M I$ body mass index. $B P$ blood pressure. $H D L$ high-density lipoprotein. $L D L$ lowdensity lipoprotein. $p$ values are based on t-tests of each variable across groups (any vs. no unplanned visits). Bold: $p<.05$ 
Table 2 Generalization performance of classifiers with optimized parameters, presented as confusion matrices and balanced accuracy \pm standard deviation across five-fold cross-validation

\begin{tabular}{|c|c|c|c|c|}
\hline Classifier & $\begin{array}{l}\text { Most stable parameters across outer } \\
\text { folds }\end{array}$ & & $\begin{array}{l}\text { Predicted: no } \\
\text { unplanned } \\
\text { visits }\end{array}$ & $\begin{array}{l}\text { Predicted: } \geq 1 \text { unplanned } \\
\text { visit }\end{array}$ \\
\hline \multirow{3}{*}{ Linear discriminant analysis } & \multirow[t]{3}{*}{ N/A } & Actual: No Unplanned Visits & $50.8 \% \pm 1.4 \%$ & $49.2 \% \pm 1.4 \%$ \\
\hline & & Actual: $\geq 1$ Unplanned Visit & $24.8 \% \pm 1.0 \%$ & $75.2 \% \pm 1.0 \%$ \\
\hline & & Average & $63.0 \% \pm 0.7 \%$ & \\
\hline \multirow[t]{3}{*}{ Quadratic discriminant analysis } & \multirow[t]{3}{*}{ N/A } & Actual: No Unplanned Visits & $82.5 \% \pm 0.6 \%$ & $17.5 \% \pm 0.6 \%$ \\
\hline & & Actual: $\geq 1$ Unplanned Visit & $56.3 \% \pm 0.8 \%$ & \multirow[t]{2}{*}{$43.7 \% \pm 0.8 \%$} \\
\hline & & Average & $63.3 \% \pm 0.4 \%$ & \\
\hline \multirow[t]{3}{*}{ Linear SVM } & \multirow[t]{3}{*}{ Cost $=25$} & Actual: No Unplanned Visits & $71.1 \% \pm 0.8 \%$ & $28.9 \% \pm 0.8 \%$ \\
\hline & & Actual: $\geq 1$ Unplanned Visit & $39.8 \% \pm 1.0 \%$ & \multirow[t]{2}{*}{$60.2 \% \pm 1.0 \%$} \\
\hline & & Average & $65.7 \% \pm 0.3 \%$ & \\
\hline \multirow[t]{3}{*}{ Radial SVM } & \multirow{3}{*}{$\begin{array}{l}\text { Cost }=50 ; \\
\text { Gamma }=0.1\end{array}$} & Actual: No Unplanned Visits & $57.6 \% \pm 1.4 \%$ & $42.5 \% \pm 1.4 \%$ \\
\hline & & Actual: $\geq 1$ Unplanned Visit & $28.4 \% \pm 0.9 \%$ & \multirow[t]{2}{*}{$71.6 \% \pm 0.9 \%$} \\
\hline & & Average & $64.6 \% \pm 0.8 \%$ & \\
\hline \multirow[t]{3}{*}{ Single hidden layer NN } & \multirow{3}{*}{$\begin{array}{l}\text { Hidden layer }=20 \text { nodes; } \\
\text { Iterations }=200 ; \\
\text { Decay }=0.0\end{array}$} & Actual: No Unplanned Visits & $50.7 \% \pm 28.7 \%$ & $49.3 \% \pm 28.7 \%$ \\
\hline & & Actual: $\geq 1$ Unplanned Visit & $31.6 \% \pm 20.4 \%$ & \multirow[t]{2}{*}{$68.4 \% \pm 20.4 \%$} \\
\hline & & Average & $59.5 \% \pm 7.7 \%$ & \\
\hline \multirow[t]{3}{*}{ Triple hidden layer DNN } & \multirow{3}{*}{$\begin{array}{l}\text { Hidden layers }=20 \text { nodes; } \\
\text { Learning }=1.0 ; \\
\text { Momentum }=0.5 \\
\text { Iterations }=20\end{array}$} & Actual: No Unplanned Visits & $65.7 \% \pm 14.5 \%$ & $34.4 \% \pm 14.5 \%$ \\
\hline & & Actual: $\geq 1$ Unplanned Visit & $36.7 \% \pm 14.6 \%$ & \multirow[t]{2}{*}{$63.3 \% \pm 14.6 \%$} \\
\hline & & Average & $64.5 \% \pm 0.8 \%$ & \\
\hline \multirow[t]{3}{*}{ XG boost } & \multirow{3}{*}{$\begin{array}{l}\text { Max depth =20; Eta =0.90; } \# \\
\text { rounds = 200; Gamma = 10; Min. } \\
\text { child weight = 10; Ratio of column } \\
\text { per tree= }=1.0\end{array}$} & Actual: No Unplanned Visits & $33.9 \% \pm 30.8 \%$ & \multirow{3}{*}{$\begin{array}{l}66.1 \% \pm 30.8 \% \\
83.3 \% \pm 15.3 \%\end{array}$} \\
\hline & & Actual: $\geq 1$ Unplanned Visit & $16.7 \% \pm 15.3 \%$ & \\
\hline & & Average & $58.6 \% \pm 7.8 \%$ & \\
\hline \multirow[t]{3}{*}{ Logistic Regression } & \multirow[t]{3}{*}{ N/A } & Actual: No Unplanned Visits & $60.4 \% \pm 0.8 \%$ & $39.6 \% \pm 0.8 \%$ \\
\hline & & Actual: $\geq 1$ Unplanned Visit & $29.8 \% \pm 0.8 \%$ & $70.2 \% \pm 0.8 \%$ \\
\hline & & Average & $65.3 \% \pm 0.7 \%$ & \\
\hline
\end{tabular}

Basic cross-validation was run for classifiers without hypermarameters (linear and quadratic discriminant analysis, logistic regression) and nested cross-validation for classifiers with hyperparameters (linear and radial SVM, single- layer NN and triple-layer DNN) to optimize hyperparameters

Cross-validation matrices show the generalization performance with respect to the actual class (rows) against the predicted class (columns), with \pm standard deviation across cross-validation runs. DNN deep nets. NN neural nets. SVM support vector machines. XG boost extreme gradient boosting

a fraction of a percentage point in most cases), which is one indicator of a low degree of overfitting [24].

Table 5 shows the sensitivity analysis of the optimized linear SVM classifier presented in Table 2, using the optimized model to predict on each subset with normally-distributed noise added to each variable, one at a time. Both blood pressure and HDL cholesterol were found to contribute most significantly to the prediction task: adding noise to the blood pressure variable (thus disrupting its contribution to the prediction task) decreased the model's balanced accuracy by 1.3 percentage points, and adding noise to the HDL cholesterol variable resulted in a decrease of 1.4 percentage points. The smallest change in balanced accuracy comes from LDL which when removed from the model, did not disrupt balanced accuracy at all. These analyses show that BP and HDL seem to be the most important indicators of unplanned medical visits among patients with diabetes, among the potentially modifiable variables.

The above findings were among many components that led to the development of a clinical intervention at the sponsoring healthcare organization. Faced with time limitations and the need to provide evidence-based recommendations to inform an intervention, the research team and the organization made the choice to forego further refinements to the predictive model and proceed with the recommendations above (i.e. targeting HDL and BP, among other factors decided by other participants in the larger process).

Moreover, practical and patient-centered considerations outweigh further gains in predictive accuracy when delivering an intervention: for example, patients often have difficulty comprehending numerical risk presented to them $[25,26]$. Thus, a highly accurate model will unfortunately be ineffective if the risk is not communicated to 
Table 3 Optimal hyper-parameters across each of 5 "outer" folds in nested cross-validation

\begin{tabular}{|c|c|c|c|c|c|}
\hline Parameter & Fold 1 & Fold 2 & Fold 3 & Fold 4 & Fold 5 \\
\hline \multicolumn{6}{|l|}{ Linear SVM } \\
\hline Cost & 0.1 & 25 & 25 & 25 & 25 \\
\hline \multicolumn{6}{|l|}{ Radial SVM } \\
\hline Cost & 25 & 50 & 50 & 50 & 50 \\
\hline Gamma & 0.1 & 0.1 & 0.1 & 0.1 & 0.1 \\
\hline \multicolumn{6}{|l|}{ Single-layer NN } \\
\hline Size of hidden layer & 15 & 20 & 20 & 20 & 1 \\
\hline Maximum \# iterations & 100 & 200 & 200 & 200 & 100 \\
\hline Decay & 0.0 & 0.0 & 0.0 & 0.0 & 0.1 \\
\hline \multicolumn{6}{|l|}{ Triple-layer DNN } \\
\hline Size of 3 hidden layers & $20,20,20$ & $20,20,20$ & $20,20,20$ & $20,20,20$ & $20,20,20$ \\
\hline Learning rate & 1 & 1 & 1 & 1 & 1 \\
\hline Momentum & 0.5 & 0.5 & 0.5 & 0.5 & 0.5 \\
\hline Number of epochs & 20 & 20 & 20 & 20 & 20 \\
\hline \multicolumn{6}{|l|}{ XG Boost } \\
\hline Max depth & 20 & 20 & 6 & 20 & 6 \\
\hline Eta & 0.9 & 0.9 & 0.01 & 0.9 & 0.01 \\
\hline Nrounds & 200 & 200 & 50 & 200 & 50 \\
\hline Gamma & 10 & 10 & 0 & 10 & 0 \\
\hline Min. child weight & 10 & 10 & 0 & 10 & 0 \\
\hline Ratio of columns per tree & 1.0 & 1.0 & 0.1 & 1.0 & 0.1 \\
\hline
\end{tabular}

NN neural nets. DNN deep nets. SVM support vector machines. XG boost extreme gradient boosting

patients in a way that they are able to understand and which would motivate behavioral changes. For this reason, the importance of improving the predictive model's balanced accuracy became less pressing than implementation science considerations. Our research team then turned to the behavioral economics literature [27-29] to identify best practices for enrolling patients and maintaining participation in the eventual intervention.

Also important is stakeholder engagement; for an intervention to be successful, it must have the buy-in of multiple participating sectors [30,31], including physicians, staff, and clinical operations. Physicians and healthcare staff were consulted with to understand how our model's recommendations fit with their current standard of care and what new steps they would be willing to take in the clinic. For example, most physicians faced with patients with diabetes or pre-diabetes are already doing everything they can to improve cholesterol levels and lower BP. The operations sector was also consulted in order to identify how to streamline the intervention most easily into the existing workflows, and how to most efficiently collect essential process and outcome data while minimizing increases to provider workload. After this stakeholder engagement process, a behavioral intervention was decided on which involves shared decision-making between providers and patients to pursue one of 5 behavioral changes: weight loss; increased physical activity; nutrition counseling; smoking cessation; and medication. This intervention is currently being piloted at the sponsoring organization.

\section{Discussion}

This study utilized machine learning to predict unplanned medical visits among patients with diabetes over a 3-year period, using readily available variables from EMRs as prediction variables. Linear-basis SVM was able to achieve slightly but significantly more accurate prediction relative to conventional logistic regression, with average balanced accuracy (average of sensitivity and specificity) of $65.7 \%$, representing a 0.4 percentage point increase over logistic regression. Further, post-hoc analysis of the optimized prediction model revealed that HDL and $\mathrm{BP}$ are possibly the most important modifiable variables that predict unplanned medical visits among patients with diabetes. These recommendations from the predictive modeling were one of many components that led to the development of a clinical intervention now being piloted at the sponsoring healthcare organization.

HDL and BP may be driving unplanned medical visits among patients with diabetes due to their individual risks for unplanned medical. HDL is generally known as 
Table 4 Training performance of classifiers with optimized parameters, presented as confusion matrices and balanced accuracy \pm standard deviation across five-fold cross-validation runs

\begin{tabular}{ll}
\hline Classifier & $\begin{array}{l}\text { Predicted: no } \\
\text { unplanned } \\
\text { visits }\end{array}$
\end{tabular}

\begin{tabular}{|c|c|c|c|}
\hline \multicolumn{4}{|c|}{ Linear discriminant analysis } \\
\hline $\begin{array}{l}\text { Actual: No Unplanned } \\
\text { Visits }\end{array}$ & $50.7 \% \pm 1.1 \%$ & \multicolumn{2}{|l|}{$49.3 \% \pm 1.1 \%$} \\
\hline $\begin{array}{l}\text { Actual: } \geq 1 \text { Unplanned } \\
\text { Visit }\end{array}$ & $24.7 \% \pm 0.7 \%$ & \multicolumn{2}{|l|}{$75.3 \% \pm 0.7 \%$} \\
\hline Average & $63.0 \pm 0.2 \%$ & & \\
\hline \multicolumn{4}{|c|}{ Quadratic discriminant analysis } \\
\hline $\begin{array}{l}\text { Actual: No Unplanned } \\
\text { Visits }\end{array}$ & $83.0 \% \pm 0.2 \%$ & \multicolumn{2}{|l|}{$17.1 \% \pm 0.2 \%$} \\
\hline $\begin{array}{l}\text { Actual: } \geq 1 \text { Unplanned } \\
\text { Visit }\end{array}$ & $56.2 \% \pm 0.2 \%$ & \multicolumn{2}{|l|}{$43.8 \% \pm 0.2 \%$} \\
\hline Average & $63.4 \% \pm 0.1 \%$ & & \\
\hline \multicolumn{4}{|l|}{ Linear SVM } \\
\hline $\begin{array}{l}\text { Actual: No Unplanned } \\
\text { Visits }\end{array}$ & $71.3 \% \pm 0.8 \%$ & \multicolumn{2}{|l|}{$28.7 \% \pm 0.8 \%$} \\
\hline $\begin{array}{l}\text { Actual: } \geq 1 \text { Unplanned } \\
\text { Visit }\end{array}$ & $39.6 \% \pm 0.7 \%$ & \multicolumn{2}{|l|}{$60.4 \% \pm 0.7 \%$} \\
\hline Average & $65.8 \% \pm 0.1 \%$ & & \\
\hline \multicolumn{4}{|l|}{ Radial SVM } \\
\hline $\begin{array}{l}\text { Actual: No Unplanned } \\
\text { Visits }\end{array}$ & $67.0 \% \pm 1.1 \%$ & \multicolumn{2}{|l|}{$33.0 \% \pm 1.1 \%$} \\
\hline $\begin{array}{l}\text { Actual: } \geq 1 \text { Unplanned } \\
\text { Visit }\end{array}$ & $21.4 \% \pm 0.4 \%$ & \multicolumn{2}{|l|}{$78.6 \% \pm 0.4 \%$} \\
\hline Average & $72.8 \% \pm 0.1 \%$ & & \\
\hline \multicolumn{4}{|l|}{ Single hidden layer NN } \\
\hline $\begin{array}{l}\text { Actual: No Unplanned } \\
\text { Visits }\end{array}$ & $50.8 \% \pm 28.7 \%$ & \multicolumn{2}{|l|}{$49.2 \% \pm 28.7 \%$} \\
\hline $\begin{array}{l}\text { Actual: } \geq 1 \text { Unplanned } \\
\text { Visit }\end{array}$ & $31.5 \% \pm 20.2 \%$ & \multicolumn{2}{|l|}{$68.5 \% \pm 20.2 \%$} \\
\hline Average & $59.7 \% \pm 7.9 \%$ & & \\
\hline \multicolumn{4}{|l|}{ Triple hidden layer DNN } \\
\hline $\begin{array}{l}\text { Actual: No Unplanned } \\
\text { Visits }\end{array}$ & $65.4 \% \pm 15.0 \%$ & \multicolumn{2}{|l|}{$34.6 \% \pm 15.0 \%$} \\
\hline $\begin{array}{l}\text { Actual: } \geq 1 \text { Unplanned } \\
\text { Visit }\end{array}$ & $36.5 \% \pm 14.3 \%$ & \multicolumn{2}{|l|}{$63.5 \% \pm 14.3 \%$} \\
\hline Average & $64.4 \% \pm 0.8 \%$ & & \\
\hline \multicolumn{4}{|l|}{ XG boost } \\
\hline $\begin{array}{l}\text { Actual: No Unplanned } \\
\text { Visits }\end{array}$ & $38.8 \% \pm 35.3 \%$ & \multicolumn{2}{|l|}{$61.2 \% \pm 35.3 \%$} \\
\hline $\begin{array}{l}\text { Actual: } \geq 1 \text { Unplanned } \\
\text { Visit }\end{array}$ & $12.9 \% \pm 11.2 \%$ & \multirow{3}{*}{\multicolumn{2}{|c|}{$87.2 \% \pm 11.2 \%$}} \\
\hline Average & $63.0 \% \pm 11.9 \%$ & & \\
\hline \multicolumn{2}{|l|}{ Logistic regression } & & \\
\hline $\begin{array}{l}\text { Actual: No Unplanned } \\
\text { Visits }\end{array}$ & $60.4 \% \pm 0.2 \%$ & & $39.6 \% \pm 0.2 \%$ \\
\hline $\begin{array}{l}\text { Actual: } \geq 1 \text { Unplanned } \\
\text { Visit }\end{array}$ & $29.8 \% \pm 0.2 \%$ & & $70.2 \% \pm 0.2 \%$ \\
\hline Average & $65.3 \% \pm 0.2 \%$ & & \\
\hline
\end{tabular}

Table 4 (continued)

Basic cross-validation was run for classifiers without hypermarameters (linear and quadratic discriminant analysis, logistic regression) and nested crossvalidation for classifiers with hyperparameters (linear and radial SVM, singlelayer NN and triple-layer DNN) to optimize hyperparameters

Cross-validation matrices show the training performance with respect to the actual class (rows) against the predicted class (columns), with \pm standard deviation across cross-validation runs. DNN deep nets. NN neural nets. SVM support vector machines. XG boost extreme gradient boosting

Table 5 Sensitivity analysis showing the disruption of balanced accuracy when adding normally-distributed noise (0.3 $\times$ standard deviation) to each variable

\begin{tabular}{lll}
\hline Variable range & $\begin{array}{l}\text { New balanced } \\
\text { accuracy (\%) }\end{array}$ & $\begin{array}{l}\text { Change in balanced accuracy } \\
\text { (vs. 65.8\% on original sample) } \\
(\%)\end{array}$ \\
\hline A1C & 65.7 & -0.1 \\
BMl & 64.7 & -1.1 \\
BP & 64.5 & -1.3 \\
HDL & 64.4 & -1.4 \\
LDL & 65.8 & -0.0 \\
Tobacco use & 65.0 & -0.8 \\
\hline
\end{tabular}

Balanced accuracy is the average of the sensitivity and specificity rates (see text), based on test sets across 25 cross-validation tests using repeated-hold$20 \%$-out subsampling. Change in balanced accuracy is relative to the optimized classification results using the original data sample in Table 2 (65.8\%)

A1C glycohemoglobin. BMI body mass index. BP blood pressure. $H D L$ highdensity lipoprotein. $L D L$ low-density lipoprotein

being the "good cholesterol" because of its atherogenesis inhibitory properties. In addition, HDL is normally antiinflammatory; however, HDL often has a loss of function in patients with diabetes, and thus the anti-inflammatory properties are inhibited [32, 33]. The disease mechanisms in both diabetes and hypertension are similar, and have commonalities in etiology including obesity, inflammation, oxidative stress, and insulin resistance [34]. Similarly, high BP in diabetes patients is associated with increased risk of death and diabetes-related complications, which explains the finding that high BP is especially predictive of unplanned medical visits [35].

Presently, literature shows more evidence of hospitals employing predictive analytics related to reducing emergency care utilization. Though not exactly comparable to the current study's aim of predicting unplanned medical visits among patients with diabetes, similar applications such as the HOSPITAL and LACE screening tools predict emergency room readmission risk. The HOSPITAL score uses seven clinical predictors to help identify patients at high risk of hospital readmissions within 30 days of discharge. This score has been validated and shown to have superior discriminative ability over other prediction tools [36]. Similarly, the LACE index uses only four variables to predict death or 30-day readmission 
after hospital discharge of $66.3 \%$ and a correct rejection rate of 53.3\% [37]. While this tool has also been validated, LACE has been shown to only have moderate discriminative ability [36]. This demonstrates the utility of such predictive models, including the current study's model for predicting unplanned medical visits among patients with diabetes.

Therefore, the higher prediction balanced accuracy in the current model demonstrates the utility of machine learning approaches for prediction of medical risks. Though the improvement in balanced accuracy may be considered small ( $\sim 0.4$ percentage points), this difference was statistically significant and could have substantial implications at a large scale. For example, back-of-the envelope calculations show that, under the assumption that these visits can be anticipated and prevented with perfect accuracy, an improvement of $0.4 \%$ for a population of 1 million patients with diabetes, given an unplanned visit rate of $57.2 \%$ (based on this sample), translates into approximately 2300 people and 7500 visits that could be avoided.

The higher accuracy is likely attributable to the increased predictive information contained in patterns of variables, over and above each variable's statistically independent association with the outcome [11,38]. Though this pattern-based information is difficult to extract in "black-box" models (e.g. SVM), we present a form of sensitivity analysis that estimates each variable's total contribution to the model (accumulated across its statistically independent main effect and all interactions with other predictor variables) and thus can quantify each variable's "diagnostic information."

Identifying the most salient predictors is an important step towards moving this predictive algorithm into concrete implementation in clinical settings. That is, the trained predictive model can be used for hypothesis generation (i.e. that risky HDL and BP values lead to unplanned medical visits). Since the predictive model itself cannot test or establish causality, further longitudinal research in clinical settings is needed to test these hypotheses; nevertheless, this hypothesis generation is an essential step in that it reduces the number of likely hypotheses that must be tested in clinical settings, leading to a more efficient use of resources. Following the hypothesis validation stage, an evidence-based intervention then can be designed and implemented which flags high-risk patients for an appropriate protocol (e.g. more aggressive targeting of BP and HDL through clinical or behavioral measures).

The above recommendations were one component of many in the development of a clinical intervention for at-risk patients, along with physician engagement, interfacing with clinical operations, and utilizing behavioral economics to maximize patient engagement with the intervention. Practical limitations in this project are common to many other projects which seek to translate research into clinical practice; most notably in the current study, the trade-off between focusing efforts on improving model accuracy versus focusing efforts on implementation science to ultimately maximize patient engagement. Thus, this study demonstrates the value of this study's approach not only in improved prediction of costly unplanned medical visits, but also in moving towards clinical implementation.

\section{Limitations}

This study has several limitations. First, causality cannot be established using observational data; however, the current procedure of performing a sensitivity analysis on modifiable predictor variables produces a more refined set of causal hypotheses that can be pursued in followup research. A related limitation is that factors that may be relevant for prediction may not be pertinent for treatment (e.g. age which is not modifiable). Additionally, results may not be generalizable to other populations outside the North Dakota, South Dakota, and Minnesota, and further validation is needed in other independent samples.

Other methodological limitations exist which, if rectified, could improve models' balanced accuracy and predictive power. Specifically, the types of classifiers used here are not comprehensive, and other methods such as random forests could offer improvements in predictive accuracy. Within the existing classifiers, it is also possible that optimizing across much wider parameter ranges could lead to higher balanced accuracy. When taking these methodological limitations into account, the marginal gains of model accuracy must be weighed against practical considerations if being used in clinical settings.

Limitations of EMR data are numerous, and this is unfortunately common when using EMR data for research. The data available had limited variables, such as imprecise measures of smoking status; and the unavailability of other variables including socio-economic and behavioral determinants of health, disease duration and severity, and depression in the current EMR system, are likely to negatively impact the prediction accuracy. However, basing our prediction model on standard EMR fields increases its utility within this healthcare system, as well as its potential generalizability of these methods to other healthcare systems. The current data were also limited with respect to the granularity of time-varying variables (e.g. BMI, BP): namely, in the dataset provided by the sponsoring healthcare organization, only the last time point was provided. This is problematic because for true prediction, the predictors must precede the outcome 
in time. However, many of these factors are fairly stable over time, lessening the impact of this limitation. Further, this and other data limitations are common in health services research, and this study provides a practical example of how clinical implications can be generated from a predictive model in spite of realistic data limitations. Another limitation is that the outcome variable of unplanned medical visits does not consider the cause of the visit; thus, visits may or may not be related to diabetes. Though there is some literature on identifying preventable emergency visits, this is a difficult process, and to our knowledge no method exists for identifying diabetes-attributable visits. Therefore, we analyze all unplanned visits together, which represents a realistic situation when analyzing EMR data. These data limitations are common for administrative health records; thus, the current study is practical in the sense that it is representative of working with real-world data limitations. However, more rigorous data with fewer of the above limitations can improve predictive modeling. Thus, improvements to data collection and querying processes and capabilities should be a priority for the use of administrative health records in research.

\section{Strengths}

The use of EMR data from a large healthcare system in the US allows for the capture of large proportion of the population, and a large sample size. This study also utilizes innovative machine learning methods with crossvalidation, which leads to improved prediction accuracy and generalizability of results. Finally, the current study demonstrates a relatively novel procedure for moving a machine-learning model from pure prediction towards making clinical improvements to care management.

\section{Conclusions}

This study shows improved prediction of unplanned medical visits among patients with diabetes by utilizing machine learning methods, relative to conventional prediction models. A post-hoc sensitivity analysis identified low HDL and high BP as the strongest predictors of unplanned medical visits among this patient population, warranting future research in clinical settings on whether these are causal relationships. Future research is underway based on this predictive model on a behavioral health intervention aimed at improving diabetes management in clinical settings. Improvements are needed to standard data collection and querying procedures for administrative health records in order to overcome important data limitations that limit current predictive modeling.

\section{Abbreviations}

EMR: Electronic medical records; BMI: Body mass index; BP: Blood pressure; LDL: Low-density lipoprotein; HDL: High-density lipoprotein; A1C: Glycohemoglobin; MN: Minnesota; ND: North Dakota; SD: South Dakota; SVM: Support vector machine; NN: Neural network; XG boost: Extreme gradient boosting.

\section{Acknowledgements}

The authors would like to thank Floe Foxon for assistance with literature and references related to methodological concepts, and Courtney Brackin for support with document preparation and editing.

\section{Authors' contributions}

All authors contributed to the production of this study and the manuscript. AS and EG designed the study. AS and DA performed data analyses. TW and CW drafted the manuscript. BS revised the document for intellectual content. All authors have read and approved the final manuscript.

\section{Funding}

This work was supported by an award from the Sanford Data Collaborative, and by the National Institute for General Medical Sciences (NIGMS), Grant No. 1P20GM121341-01. The funders had no role in study design, data collection and analysis, decision to publish, or preparation of the manuscript.

\section{Availability of data and materials}

The datasets used during the current study can be applied for through the Sanford Data Collaborative: https://research.sanfordhealth.org/services/sanfo rd-data-collaborative. The code used in this study is freely available at: https:// github.com/ArielleSelya/Diabetes-Predictive-Model.

\section{Declarations}

Ethics approval and consent to participate

This study was approved by Sanford Research's Internal Review Board. Permission from Sanford Research's Privacy Board and Internal Review Board were required to access the data used in this study.

\section{Competing interests}

The authors declare that they have no competing interests.

\section{Consent for publication}

Not applicable.

\section{Author details}

${ }^{1}$ Department of Population Health, University of North Dakota School of Medicine and Health Sciences, Grand Forks, ND, USA. ${ }^{2}$ Behavioral Sciences Group, Sanford Research, 2301 East 60th Street North, Sioux Falls, SD 57104, USA. ${ }^{3}$ Department of Pediatrics, University of South Dakota Sanford School of Medicine, Sioux Falls, SD, USA. ${ }^{4}$ Pinney Associates, Inc., Pittsburgh, PA, USA.

${ }^{5}$ Advanced Analytics, St. Luke's Health System, Boise, ID, USA. ${ }^{6}$ Sanford Heath Plan, Sioux Falls, SD, USA. ${ }^{7}$ EDCO Health Information Systems, Sioux Falls, SD, USA.

Received: 3 September 2020 Accepted: 22 March 2021

Published online: 31 March 2021

\section{References}

1. National Diabetes Statistics Report, 2020 | CDC. 2020. https://www.cdc gov/diabetes/data/statistics-report/index.html. Accessed 30 Dec 30.

2. Rui P, Kang K, Ashman J. National hospital ambulatory medical care survey: 2016 emergency department summary tables. 2016.

3. American Diabetes Association. Economic Costs of Diabetes in the U.S. in 2017. Diabetes Care 2018;41. https://doi.org/10.2337/dci18-0007.

4. Raghupathi W, Raghupathi V. An empirical study of chronic diseases in the United States: a visual analytics approach to public health. Int J Environ Res Public Health. 2018. https://doi.org/10.3390/ijerph1503 0431. 
5. Bodenheimer T, Chen E, Bennett HD. Confronting the growing burden of chronic disease: can the US health care workforce do the job? Health Aff Proj Hope. 2009;28:64-74. https://doi.org/10.1377/hlthaff.28.1.64.

6. McEwen LN, Herman WH. Health care utilization and costs of diabetes. Diabetes in America. 3rd ed. Bethesda: National Institutes of Health; 2018. p. 40-1-40-78.

7. Druss BG, Marcus SC, Olfson M, Tanielian T, Elinson L, Pincus HA. Comparing the national economic burden of five chronic conditions. Health Aff Proj Hope. 2001:20:233-41. https://doi.org/10.1377/hlthaff.20.6.233.

8. Begum N, Donald M, Ozolins IZ, Dower J. Hospital admissions, emergency department utilisation and patient activation for self-management among people with diabetes. Diabetes Res Clin Pract. 2011;93:260-7. https://doi.org/10.1016/j.diabres.2011.05.031.

9. Selya A, Johnson EL, Weber TL, Russo J, Stansbury C, Anshutz D, et al. Smoking is associated with a higher risk of unplanned medical visits among adult patients with diabetes, using retrospective electronic medical record data from 2014 to 2016. BMC Health Serv Res. 2020. https://doi. org/10.1186/s12913-020-05277-4.

10. Deo RC. Machine learning in medicine. Circulation. 2015;132:1920-30. https://doi.org/10.1161/CIRCULATIONAHA.115.001593.

11. Selya AS, Anshutz D. Machine learning for predicting health outcomes: An example of predicting obesity from dietary and physical activity patterns. Adv. Data Anal. Healthc., Switzerland: Springer; 2018, pp. 77-97.

12. Giabbanelli PJ, Adams J. Identifying small groups of foods that can predict achievement of key dietary recommendations: data mining of the UK National Diet and Nutrition Survey, 2008-2012. Public Health Nutr. 2016:19:1543-51. https://doi.org/10.1017/S1368980016000185.

13. Karimi-Alavijeh F, Jalili S, Sadeghi M. Predicting metabolic syndrome using decision tree and support vector machine methods. ARYA Atheroscler. 2016;12:146-52.

14. Crutzen R, Giabbanelli PJ, Jander A, Mercken L, de Vries H. Identifying binge drinkers based on parenting dimensions and alcohol-specific parenting practices: building classifiers on adolescent-parent paired data. BMC Public Health. 2015;15:747. https://doi.org/10.1186/ s12889-015-2092-8.

15. Crutzen R, Giabbanelli P. Using classifiers to identify binge drinkers based on drinking motives. Subst Use Misuse. 2014;49:110-5. https://doi.org/10. 3109/10826084.2013.824467.

16. Golino HF, Amaral LSDB, Duarte SFP, Gomes CMA, Soares TDJ, DosReis LA, et al. Predicting increased blood pressure using machine learning. J Obes. 2014;2014:e637635. https://doi.org/10.1155/2014/637635.

17. R Core Team. R: A language and environment for statistical computing. Vienna, Austria: R Foundation for Statistical Computing; 2018.

18. Venables WN, Ripley BD. Modern applied statistics with S. 4th ed. New York: Springer; 2002. https://doi.org/10.1007/978-0-387-21706-2.

19. Meyer D, Dimitriadou E, Hornik K, Weingessel A, Leisch F, C++-code) C-CC (libsvm, et al. e1071: Misc Functions of the Department of Statistics, Probability Theory Group (Formerly: E1071), TU Wien. 2019.

20. Rong X. deepnet: deep learning toolkit in R. 2014.

21. Chen T, He T, Benesty M, Khotilovich V, Tang Y, Cho H, et al. Extreme Gradient Boosting. R package version 1.3.2.1. 2021.

22. Cawley GC, Talbot NL. On over-fitting in model selection and subsequent selection bias in performance evaluation. J Mach Learn Res. 2010;11:2079-107.
23. Kaivanto K. Maximization of the sum of sensitivity and specificity as a diagnostic cutpoint criterion. J Clin Epidemiol. 2008;61:517-8. https://doi. org/10.1016/j.jclinepi.2007.10.011.

24. Mehrvar M, Rostamizadeh A, Talwalkar A. Foundations of machine learning. The MIT Press; 2018.

25. Edwards A, Elwyn G, Mulley A. Explaining risks: turning numerical data into meaningful pictures. BMJ. 2002;324:827-30. https://doi.org/10.1136/ bmj.324.7341.827.

26. Wilhelms EA, Reyna VF. Effective ways to communicate risk and benefit. AMA J Ethics. 2013;15:34-41. https://doi.org/10.1001/virtualmentor.2013. 15.1.stas1-1301.

27. Thaler $\mathrm{RH}$, Sunstein CR. Nudge: improving decisions about health, wealth, and happiness. New York: Penguin Books, Ltd.; 2009.

28. Kahneman D, Tversky A. Prospect theory: an analysis of decision under risk. Econometrica. 1979;47:263-91. https://doi.org/10.2307/1914185.

29. Zimmerman FJ. Using behavioral economics to promote physical activity. Prev Med. 2009;49:289-91. https://doi.org/10.1016/j.ypmed.2009.07.008.

30. Bastian LA, Cohen SP, Katsovich L, Becker WC, Brummett BR, Burgess DJ, et al. Stakeholder engagement in pragmatic clinical trials: emphasizing relationships to improve pain management delivery and outcomes. Pain Med Malden Mass. 2020;21:S13-20. https://doi.org/10.1093/pm/pnaa333.

31. Kim KK, Khodyakov D, Marie K, Taras H, Meeker D, Campos HO, et al. A novel stakeholder engagement approach for patient-centered outcomes research. Med Care. 2018;56:S41-7. https://doi.org/10.1097/MLR.00000 00000000790.

32. Femlak M, Gluba-Brzózka A, Ciałkowska-Rysz A, Rysz J. The role and function of HDL in patients with diabetes mellitus and the related cardiovascular risk. Lipids Health Dis. 2017. https://doi.org/10.1186/ s12944-017-0594-3.

33. Farbstein D, Levy AP. HDL dysfunction in diabetes: causes and possible treatments. Expert Rev Cardiovasc Ther. 2012;10:353-61. https://doi.org/ 10.1586/erc.11.182.

34. Cheung BMY, Li C. Diabetes and hypertension: is there a common metabolic pathway? Curr Atheroscler Rep. 2012;14:160-6. https://doi.org/10. 1007/s11883-012-0227-2.

35. Group BMJP. Tight blood pressure control and risk of macrovascular and microvascular complications in type 2 diabetes: UKPDS 38. BMJ. 1998;317:703-13. https://doi.org/10.1136/bmj.317.7160.703.

36. Robinson R, Hudali T. The HOSPITAL score and LACE index as predictors of 30 day readmission in a retrospective study at a university-affiliated community hospital. PeerJ. 2017. https://doi.org/10.7717/peerj.3137.

37. Low LL, Lee KH, Hock Ong ME, Wang S, Tan SY, Thumboo J, et al. Predicting 30-day readmissions: performance of the LACE index compared with a regression model among general medicine patients in Singapore. BioMed Res Int. 2015;2015:169870. https://doi.org/10.1155/2015/169870.

38. Hanson SJ, Schmidt A. High-resolution imaging of the fusiform face area (FFA) using multivariate non-linear classifiers shows diagnosticity for nonface categories. Neuroimage. 2011;54:1715-34. https://doi.org/10.1016/j. neuroimage.2010.08.028

\section{Publisher's Note}

Springer Nature remains neutral with regard to jurisdictional claims in published maps and institutional affiliations.
Ready to submit your research? Choose BMC and benefit from:

- fast, convenient online submission

- thorough peer review by experienced researchers in your field

- rapid publication on acceptance

- support for research data, including large and complex data types

- gold Open Access which fosters wider collaboration and increased citations

- maximum visibility for your research: over 100M website views per year

At BMC, research is always in progress.

Learn more biomedcentral.com/submissions 